\title{
Ein Beitrag zur Frage des Pflegerunterrichtes.
}

\author{
Von \\ Dr. Tomaschny (Stralsund). \\ (Volontairarzt der Anstalt Lauenburg von August 1898 bis April 1899.)
}

(Eingegangen am 12. April 1914.)

Neben den großen wissenschaftlichen Problemen, welche in den letzten Jahren auf dem Gebiete der Psychiatrie das allgemeine Interesse für sich in Anspruch nehmen, drohen, wie uns ein Blick auf die Literatur lehrt, die rein praktischen Fragen ganz aus dem Bereiche unserer Betrachtung zu verschwinden und in Vergessenheit zu geraten. Mit Unrecht, denn für uns Anstaltsärzte hat die Beschäftigung mit den praktischen Aufgaben, welche die Versorgung der Kranken mit sich bringt, mindestens die gleiche Bedeutung wie das Studium der verschiedenen, meist noch im Ungewissen und Dunklen sich bewegenden Theorien über das Wesen und die Entstehung einzelner Psychosen, und es ist durchaus notwendig, daß auch den praktischen Fragen unsere Aufmerksamkeit dauernd gewidmet bleibt. $\mathrm{Zu}$ diesen gehört nicht an letzter Stelle die Ausbildung unseres Pflegepersonals, worüber in den letzten 4 bis 5 Jahren wenig zu hören war, obwohl eine vollkommen befriedigende Lösung dieser Frage durchaus noch nicht gefunden ist. Darüber, daß die Ausbildung unseres Personals durch Erteilung eines systematischen Unterrichtes gefördert werden muß, dürfte jetzt wohl so ziemlich allgemeine Übereinstimmung herrschen. Vor nicht allzulanger Zeit war das noch nicht der Fall. Eine im Jahre 1896 von der Prov.-Heilanstalt zu La uenburg i. Pom. ausgehende Rundfrage ergab, daß damals unter 65 öffentlichen Anstalten Deutschlands, Österreichs, Hollands und der Schweiz erst 26, also noch nicht die Hälfte, regelmäßige Wärterkurse abhielten; während ungefähr 10 Jahre später Starlinger feststellen konrite, daß unter 78 Anstalten derselben Länder nur noch 9 keinen Unterricht erteilten, und es ist wohl mit Sicherheit anzunehmen, daß inzwischen auch diese wenigen Anstalten sich der allgemeinen Auffassung von der Notwendigkeit regelmäßiger Pflegerkurse angeschlossen haben. Große Meinungsverschiedenheit herrscht dagegen anscheinend noch über die Art der Handhabung und Ausgestaltung des Unterrichtes, und doch ist es sehr erwünscht, daß auch in diesen Punkten möglichste thbereinstimmung erzielt wird. Hierzu ist es notwendig, daß die überall gemachten Erfahrungen mitgeteilt 
werden und zu gegenseitigem Gedankenaustausch anregen. In Po m mern wird schon seit dem Jahre 1893 regelmäßiger Pflegerunterricht erteilt, und zwar waren es besonders Siemens und Mercklin, welche das Interesse für diese Frage dauernd rege erhielten und selbst an ihrer Lösung eifrig mitarbeiteten. Nachdem ich nun auch seit mehr als 15 Jahren in derselben Provinz meine Aufmerksamkeit dem gleichen Gegenstande gewidmet habe, möchte ich im folgenden an der Hand der eigenen Erfahrungen sowie unter gleichzeitiger Berücksichtigung der anderwärts herrschenden Verhältnisse und der von anderen Seiten geäußerten Anschauungen mitteilen, wie ich mir eine zweck mäßige Ausgestaltung der Pflegerkurse denke.

$\mathrm{Zu}$ Beginn dieser Besprechungen sei mit einigen Worten darauf eingegangen, ob in einer Anstalt beim Bestehen regelmäßiger Pflegerkurse das vorhandene Personal ausnahmslos zur Teilnahme an diesem Unterricht gezwungen sein soll. Ich würde es eigentlich für überflüssig halten, die Frage überhaupt aufzuwerfen, wenn ich nicht tatsächlich hier und da der Auffassung begegnet wäre, daß man es ruhig dem einzelnen überlassen solle, ob er an dem Unterricht teilnehmen will oder nicht. Meiner Meinung nach kann es gar keinem Zweifel unterliegen, daß der Unterricht für alle Pflegepersonen ohne Ausnahme obligatorisch sein muß. Abgesehen von vielen anderen Gründen, welche für diese Notwendigkeit sprechen, werden wir hierdurch am besten die in Laienkreisen noch vielfach verbreitete Ansicht zerstreuen, als ob jeder Mensch so ohne weiteres, gleichsam von der Straße kommend, geeignet wäre, Geisteskranke zu behüten und zu pflegen. Das neu eintretende Personal aber wird durch diese Verpflichtung erkennen lernen, daß es jetzt einem Berufe angehört, der wie jeder andere Beruf erst mit Eifer und Fleiß erlernt und studiert werden muß. Das italienische Irrengesetz vom 5. März 1905 bestimmt ausdrücklich, daß jeder Pfleger an einem solchen Unterricht teilgenommen und auch ein Examen abgelegt haben muß. Um allen Weiterungen aus dem Wege zu gehen, empfiehlt es sich, gleich bei der Einstellung jede Pflegeperson auf die Pflicht zur Teilnahme an dem Kursus hinzuweisen. $\mathrm{Ob}$ es notwendig ist, bis zur Heranziehung zum Unterricht eine bestimmte Wartezeit vorzuschreiben, erscheint mir fraglich; auch dürfte sich eine solche Vorschrift, wenigstens bei unseren Verhältnissen, praktisch kaum immer durchführen lassen. In einzelnen Staaten Nordamerikas wird erst nach einer vierwöchigen Probedienstzeit über die Zulassung zu den Unterrichtskursen entschieden; in Niederösterreich geschieht dies sogar erst nach Ablauf eines vollen Dienstjahres. Bei uns wird jeder Pfleger ohne Rücksicht auf die seit seinem Eintritt verstrichene Zeitdauer bei der ersten sich bietenden Gelegenheit zum Kursus herangezogen. Während des Unterrichtes lernt ja 
der Arzt, bei richtiger Handhabung desselben, die Pflegepersonen viel besser kennen als auf der Abteilung, und es wird ihm dann schon nach kurzer Zeit möglich sein, die unbrauchbaren Elemente herauszufinden und rechtzeitig zum Ausscheiden zu bringen. Um allzu ungebildetes Personal vom Pflegeberufe fernzuhalten, empfiehlt es sich, die einzelnen beim Eintritt einer kurzen Prüf ung in den Elementarsch ulkenntnissen zu unterziehen, eine Einrichtung, die wie ich sehe, bereits in Victoria besteht.

Für die Erteilung des Unterrichtes ist unumgänglich notwendig ein eigenes Unterrichtszimmer. In einigen Anstalten wird, wie ich aus der oben erwähnten Rundfrage Starlingers entnehme, das Personal auf den Abteilungen gelegentlich der Visiten unterwiesen; diese Art des Unterrichts allein erachte ich für ungenügend. Hierbei können fortwährend allerlei Unterbrechungen und unerwünschte Störungen dazwischen kommen, die sich beim. Vorhandensein eines eigenen, diesem Zweck dienenden Zimmers vermeiden lassen. Ein solches Zimmer muß auch seiner Bestimmung entsprechend ausgestattet sein. Wir müssen hier anatomische Wandtafeln vorfinden, eine Schultafel, ein menschliches Skelett, wenigstens einige Modelle von den wichtigsten Teilen des menschlichen Körpers, ferner die gebräuchlichsten ärztlichen Instrumente, die verschiedenen Arten von Arzneigläsern u. dgl. Sehr gut ist auch, wenn die Mittel es gestatten, ein kleiner Projektionsapparat. In Lauenburg wurden hiermit unter anderem die verschiedensten Parasiten gezeigt, und in Herzberge werden, wie Moeli in der Sitzung des psychiatrischen Vereins zu Berlin am 13. Dezember 1902 berichtete, auf diese Weise den Pflegern zunächst falsche, dann richtige Stellungen.und Handgriffe bei Ausübung der Krankenpflege vorgeführt. In Pommern sind in den jährlichen Etat einer jeden Anstalt 100 Mark für Pflegerunterrichtszwecke eingestellt, und mit dieser Summe läßt sich im Laufe der Jahre eine recht ansehnliche Sammlung von Lehrmittelgegenständen anlegen. Außerdem muß aber jede Anstalt bestrebt sein, sich eine Art kleines $M$ use u m anzulegen, das allerhand sonstige Gegenstände birgt, die auf die Pflege Geisteskranker Bezug haben und von den Kranken selbst reichlich geliefert werden. Hierher gehören z. B. die verschiedenen oft mit großer Kunstfertigkeit hergestellten Dietriche oder waffenähnlichen Werkzeuge, die zu Angriffen auf Arzt und Personal oder auch zu Selbstrerletzungen gedacht sind; ferner die aus allem möglichen Material hergestellten Stricke zu Erhängungs- oder Fluchtversuchen u. dgl. Indem wir uns in der geschilderten Weise das Unterrichtszimmer ausstatten, werden wir nicht nur uns selbst die Freude am Unterrichten erhöhen, sondern auch beim Personal eine größere Meinung von der Bedeutung der ganzen Sache erwecken. 
Darüber, daß die Erteilung des Unterrichtes vollständig in der Hand des Arztes liegen muß, dürfte wohl im allgemeinen kein Zweifel bestehen. Aus einem Anstaltsberichte ersehe ich, daß in der betreffenden Anstalt einiges vom Oberpflegepersonal gelehrt wird, wie der Umgang mit den Kranken, die Pflege der Bettlägerigen, die Zubereitung der Bäder u. ähnl. Ich halte dieses Verfahren für unrichtig, aber nicht etwa, weil ich etwa unser Oberpflegepersonal, das im allgemeinen alle Hochachtung verdient, zur Unterweisung in einigen praktischen Handgriffen für unfähig hielte; der Grund liegt für mich vielmehr darin, daß das Personal die Wichtigkeit des Gelehrten unwillkürlich nach der persönlichen Stellung des Lehrenden einschätzen wird. Es könnte also leicht zu der Auffassung kommen, daß dem lediglich vom Oberpflegepersonal gelehrten praktischen Teil der Irrenpflege eine geringere Bedeutung zukäme als dem theoretischen, und das wäre im Interesse unserer Kranken sehr zu bedauern. Namentlich die Unterweisung im Umgang mit den Kranken sollte doch der Arzt unter keinen Umständen aus der Hand geben; gerade der Umgang mit den Kranken stellt einen wesentlichen, ja fast den wichtigsten Teil unserer Behandlung dar, er ist eine höchste Kunst, und in der Art des Umganges und dem sich hieraus ergebenden Einfluß auf die Kranken liegt ja eigentlich die ganze Bedeutung der Persönlichkeit des Irrenarztes. Ich möchte mich also ganz entschieden gegen eine reinliche Teilung des Lehrstoffes zwischen Arzt und Oberpflegepersonal aussprechen; wohl aber halte ich es für sehr nützlich und durchaus unbedenklich, daß das Oberpflegepersonal den Arzt bei Erteilung des praktischen Unterrichtes unterstützt und nach seiner Anweisung hierbei ergänzend mitwirkt. Unter den vorhandenen Ärzten einer Anstalt wird man natürlich in der Regel die älteren mit der Erteilung des Unterrichtes betrauen, doch muß hierbei immer möglichst freie Vereinbarung vorbehalten bleiben; denn es kann jemand ein sehr brauchbarer und gewissenhafter Arzt und doch ein wenig geeigneter Lehrer sein.

Eine besondere Honorierung für Erteilung des Unterrichts, wie sie von manchẻn Seiten erstrebt wird und zum Teil auch schon üblich ist, halte ich nicht für durchaus erforderlich. Mir hat das Unterrichten stets Freude gemacht, und ich sah darin immer mehr eine angenehme Abwechselung im eintönigen Anstaltsdienste als eine Last. Daß der Direktor selbst den Unterricht erteilen soll, ist eine zuweit gehende Forderung, die sich wohl nur noch in ganz kleinen Anstalten erfüllen ließe. Höchstens käme in Betracht, daß der Direktor zu Beginn der Unterrichtsperiode einen einleitenden Vortrag hält, um auch dadurch in den Augen des Personals der ganzen Einrichtung eine besondere Bedeutung zu geben.

Was die Methode des Unterrichtens anlangt, so denke ioh mir 
diese in folgender Art: Die Vortragsweise muß möglichst einfach und schlicht sein; sie soll keine rhetorische Leistung darstellen, und jeder Anstrich von Gelehrsamkeit ist streng zu vermeiden. Das Vorgetragene ist durch passende Beispiele und Vergleiche zu beleben und durch leicht faßliche schematische Zeichnungen an der Wandtafel dem Verständnis näher zu bringen. Uberhaupt kommt es, wie überall, so natürlich auch hier vor allem darauf an, daß das Vorgetragene verstanden wird. Als unerläßlich erscheint es mir deshalb auch, daß man sich durch Wiederholen und Abfragen davon überzeugt, ob der Lehrstoff in den geistigen Besitzstand der Unterrichteten übergegangen ist. Das Personal ist auch anzuregen, über das Gehörte selbst Fragen an den Lehrer zu stellen, und bei Behandlung eines neuen Lehrstoffes muß man versuchen, die Zuhörer durch eigenes Nachdenken auf die richtige Beantwortung dieser oder jener Frage zu führen; hierdurch wird das Interesse an dem behandelten Gegenstande nicht unwesentlich erhöht. Wollten wir uns lediglich auf das Halten akademischer Vorträge beschränken und es dem guten Willen des einzelnen überlassen, ob er von dem Gehörten etwas in sich aufnehmen will oder nicht, dann würden wir, wenigstens bei unserem Personal, nicht viel Erfolge erzielen. Ich bin der Meinung, man muß den Unterricht möglichst schulmäßig gestalten und stimme durchaus nicht Scholz bei, der „das Abfragen im Unterricht vielleicht praktischer, aber wegen der verzweifelten Ähnlichkeit mit dem Schulbetrieb weniger sympathisch" findet. Wenn diese Methode, wie Scholz also selbst zugibt, praktisch ist, dann ist sie auch empfehlenswert, und ich glaube, unter einer Wärterschule stellt sich wohl jeder ganz unwillkürlich viel eher eine Einrichtung mit wirklich schulmäßigem Betriebe vor als ein akademisches Institut.

Zur Sicherung eines guten Erfolges empfiehlt es sich, daß wir uns beim Unterricht an einen bestimmten Leitfaden anlehnen, der auch jedem Teilnehmer am Kursus in die Hand gegeben wird. Wenn der Lehrplan auf diese Weise im großen ganzen festgelegt ist, dann kann selbst bei längerer Behinderung oder bei gänzlichem Ausscheiden des augenblicklichen Leiters der Unterricht ohne weiteres von einem anderen Arzt fortgeführt werden, was nicht möglich ist, wenn jeder Lehrende seinen eigenen Lehrplan hat. Auch dann, wenn etwa die Prüfung vor einer Kommission von mehreren Mitgliedern stattfindet, die selbst fragend in den Gang der Prüfung eingreifen, wird viel Ärger und manche unangenehme Utberraschung erspart bleiben, wenn durch einen Leitfaden die Grenzen der Fragestellung im allgemeinen gezogen sind. Die Benutzung eines Leitfadens soll natürlich niemanden hindern, diesen nach Belieben zu ergänzen und in einzelnen Punkten nach eigenem Ermessen umzugestalten; dies wird zuweilen infolge besonderer 
örtlicher Verhältnisse sogar notwendig sein, z. B. dort, wo auch ein Verwahrungshaus für geisteskranke Verbrecher besteht. Auf eine kritische Besprechung der vorhandenen Lehrbücher für den Pflegerunterricht einzugehen ist hier nicht der Platz. Bei der Auswahl des Leitfadens sollte man aber darauf Rücksicht nehmen, daß er genügend mit guten Abbildungen versehen ist, so da $B$ ihn der Pfleger auch schon deshalb gern in die Hand nimmt. In dieser Hinsicht könnte der bekannte Scholzsche Leitfaden, den wir sonst seit jeher zur Zufriedenheit gebrauchen, noch eine kleine Aufbesserung erfahren.

Die Benutzung eines Leitfadens erleichtert uns auch die Entscheidung über den Umfang des zu behandelnden Lehrstoffes. Hierüber gehen ja die Ansichten noch sehr weit auseinander. Während die einen gelegentliche und zwar vornehmlich praktische Unterweisungen bei den ärztlichen Visiten für ausreichend halten, lassen andere ihren Pflegern regelrechte akademische Vorlesungen halten, und zwar nicht von den Anstaltsärzten, sondern von eigens hierzu gewonnenen Dozenten. Der richtige Weg liegt, wie so oft, wohl auch hier wieder in der Mitte. Wir sollen nicht zu wenig und nicht zu viel lehren, vor allem aber nur solche Dinge, die von unserem Personal nach ihrem Bildungsgrade a uch wirklich verstanden werden können. In dieser Hinsicht entspricht der Scholzsche Leitfaden wohl allen billigerweise zu stellenden Anforderungen.

Ganz abgesehen aber davon, ob ein Leitfaden benutzt wird oder nicht, ist im allgemeinen daran festzuhalten, daß der Unterricht in einen theoretischen und einen praktischen Teil zerfallen muB, welche einander ergänzen sollen und beide als gleich notwendig und wichtig anzusehen sind. Wir können hier natürlich nicht im einzelnen alles das durchsprechen, was zweckmäßig gelehrt werden soll und was nicht; nur einige zum Teil noch strittige Punkte auf die sonst weniger hingewiesen wird, seien unter kurzer Skizzierung des bei uns üblichen Lehrganges hervorgehoben. Bei Umgrenzung des theoretischen Teiles ist $z$. B. die Ansicht vertreten worden, daß es überflüssig ist, in den Lehrplan Anatomie und Physiologie aufzunehmen. Demgegenüber sei bemerkt, daß eine Beschreibung des menschlichen Körpers - natürlich nur in ganz groben Umrissen und ein kurzer Hinweis auf die Funktion der lebenswichtigen Organe als Grundlage für die weiteren Besprechungen und praktischen Utbungen ganz unerläßlich ist. Ohne eine solche Kenntnis vermag doch niemand mit Verständnis die erste Hilfe bei plötzlichen Unglücksfällen zu leisten wie die Ausübung künstlicher Atmung, die Bekämpfung einer stärkeren Blutung u. dgl. Daß aber unser Personal in der Leistung der ersten Hilfe gründlich ausgebildet sein muß, ist doch wohl selbstverständlich. Ob man das Personal auch zu einer Sektion mit heran- 
ziehen soll, ist Geschmackssache; ich richte mich dabei ganz nach den Wünschen der Schüler. Für notwendig halte ich es nicht und erachte die Unterweisung an einem Rumpfmodell für ausreichend. An die Besprechung der Anatomie und Physiologie reiht sich zweckmäßig die Lehre von der allgemeinen Krankenpflege und daran schließt sich die besondere Irrenpflege. Bei Behandlung dieses letzten Teiles streife ich stets ganz kurz die Geschichte der Psychiatrie und ziehe einen Vergleich zwischen der früheren und der jetzigen Behandlungsweise der Geisteskranken, wobei auch die Frage der Isolierung besonders erwähnt wird. Bei der Abhandlung der geistigen Störungen genügt die Schilderung einzelner besonders hervorstechender Zustandsbilder und Symptome (Angstzustände, Krampfanfälle u. dgl.); ein näheres Eingehen auf die einzelnen Krankheitsformen ist nicht nötig.

Zu Beginn oder auch am Schluß des Kursus gebe ich dann noch eine kurze Beschreibung der Anstalt und ihrer baulichen Einrichtungen und bespreche die wichtigsten Punkte der Hausordnung. Außerdem mache ich es mir zur Pflicht, am Anfang jeder Unterrichtsstunde etwaige besondere Vorkommnisse in der Anstalt (Unglücksfälle, Entweichungen, Verfehlungen des Personals u. ähnl.) zu erörtern. Schließlich suche ich auch noch insofern erzieherisch auf das Personal einzuwirken, als ich durch entsprechende Hinweise ihr Verantwortlichkeitsgefühl und Standesbewußtsein zu erwecken und zu heben trachte; hierin sehe ich einen nicht bloß nützlichen sondern auch notwendigen Nebenzweck des Unterrichtes.

Hand in Hand mit diesen theoretischen Belehrungen muß natürlich eine Unterweisung des Personals in der praktischen Kranken pflege erfolgen, und dies geschieht am besten auf der Krankenabteilung. Diese praktischen Utbungen halte ich nicht bloß systematisch in besonders hierfür angesetzten Stunden ab, sondern ich benutze außerdem jede bei den Visiten sich bietende Gelegenheit, z. B. beim Anlegen von Verbänden u. dgl., um gerade die jüngeren Pfleger nach Möglichkeit zur Hilfeleistung und Belehrung heranzuziehen. Ich bin überhaupt der Meinung, daß der Arzt während der Visiten seine Aufmerksamkeit nicht bloß auf die Kranken, sondern auch auf die - namentlich jüngeren - Pfleger zu richten hat. Diese sind regelmäßig über die Kranken zu befragen, damit sie auf diese Weise gezwungen werden, sich mit ihren Pfleglingen eingehend zu befassen. Sehr wichtig ist auch, daß wir bei möglichst häufiger Anwesenheit auf der Abteilung das Verhalten der noch unerfahrenen Pfleger gegenüber den Kranken einer ständigen Kontrolle unterziehen; hier müssen wir bei etwaigen Fehlgriffen sofort mahnend und verbessernd eingreifen und dem Personal zeigen, wo es zufassen und wo es andererseits sich zurückhalten 
soll. Eine kurze Belehrung aus der jeweiligen Situation heraus ist hier oft wertvoller als langatmige theoretische Auseinandersetzungen. Vor allem aber müssen wir das Personal darauf achten lehren, wie der Arzt selbst mit den Kranken umgeht; wenn irgendwo, so wirkt gerade hierbei das Beispiel lebendiger und nützlicher als alle schönen Worte. Der Pfleger muß dem Arzte die Kunst des Umganges mit den Kranken absehen, genau so wie der junge Arzt wiederum dem erfahrenen Psychiater die psychische Behandlung absieht.

Fassen wir zusammen, so sind also vornehmlich zwei wichtige Gesichtspunkte bei der Aufstellung des Lehrplanes für den Pflegerunterricht zu beachten: Erstens dürfen wir weder die theoretischen Belehrungen noch die praktischen Unterweisungen in einseitiger Weise bevorzugen, es muß sich vielmehr die Ausbildung gleichmäßig nach beiden Richtungen hin erstrecken. Zweitens müssen wir uns bei Auswahl des Lehrstoffes möglichst einschränken; wir sollen nur das wirklich Notwendige, dieses aber dafür um so gründlicher lehren. Wenn sich unsere Anforderungen in solchen mäßigen Grenzen halten, dann werden etwa 30 Unterrichtsstunden einschließlich einiger Wiederholungsstunden für die Bewältigung des Stoffes genügen. Die ganze Da uer des K ursus würde demnach auf etwa 4 Monate zu veranschlagen sein, wobei wöchentlich 2 Stunden anzusetzen wären. Mehr Unterrichtsstunden als 2 in der Woche sind nicht zu erteilen, da sonst die Schüler nicht imstande sind, das Vorgetragene genügend zu verarbeiten und in ihren geistigen Besitzstand aufzunehmen. Vergleichsweise seien hier die Stunden zusammengestellt, die von einigen Autoren für die Dauer eines Kursus als zweckmäßig bezeichnet werden. Mercklin hält 17-20 Stunden für ausreichend; Scholz dehnt seinen Unterricht auf 26-30 Stunden aus; in Frankreich (Seinedepartement) dauert er 88 Stunden; in einzelnen Anstalten der Vereinigten Staaten Nordamerikas währt er zwei Jahre und in Holland sogar drei Jahre. Die Verhältnisse in den letztgenannten Ländern können wir allerdings nicht so ohne weiteres mit den unsrigen vergleichen, da dort die Organisation des Pflegerstandes auf ganz anderen Grundlagen beruht wie bei uns. In Deutschland würde bei einer dreijährigen Dauer des Kursus dieser wohl nur von sehr wenigen Mitgliedern des Pflegepersonals vollständig durchgemacht werden, denn ein dreijähriges oder noch längeres Verbleiben im Anstaltsdienste gehört bei uns - wenigstens unter den Pflegerinnen - schon zu den großen Seltenheiten.

Den Abschluß des Lehrkursus hat eine Prüfung zu bilden. Diese Prüfung muß ebenso wie die Teilnahme am Unterricht für jede Pflegeperson obligatorisch sein, und es ist am besten auch hierauf gleich bei der Einstellung in den Dienst hinzuweisen. Vielfach scheint man, wie die in der Literatur niedergelegten Anschauungen beweisen, von 
der Notwendigkeit einer solchen Schlußprüfung noch nicht überzeugt zu sein, wenigstens in Deutschland, während uns das Ausland in dieser Hinsicht weit voraus ist.

Die Jahresversammlung des Vereins deutscher Irrenärzte zu Hannover im Jahre 1897 sprach sich abweichend von dem von Siemens gemachten Vorschlage gegen die Einführung einer obligatorischen Prüfung aus. In derselben Versammlung schlug Alt vor, man solle die Prüfung ins Belieben des Personals stellen. Wickel sagt in seinem im 9. Jahrgang der Psychiatrisch-Neurologischen Wochenschrift veröffentlichten Beitrag zur Pflegerfrage: „Ein Examen findet, soviel ich weiß, in der Regel nicht statt" und in manchen Anstaltsberichten, wie z. B. aus Conradstein, findet man noch in neuester Zeit die Bemerkung, daß besondere Prüfungen nicht für erforderlich gehalten werden. Ich kann diese Ansicht nicht teilen. Es ist doch ganz sicher, daß eine in Aussicht stehende Prüfung viele zu größerem Eifer anspornt, so daß sich hierdurch das Gesamtergebnis des Unterrichtes günstiger gestaltet. Sodann wird die Prüfung auch dazu beitragen, nach außen das Ansehen des Pflegerstandes, nach innen das Standesbewußtsein zu erhöhen, ohne daß man hierbei gleich an eine ungesunde Selbstüberhebung denken muß. Zu wünschen ist nur, daß die Prüf ung nicht zu schwierig gemacht wird, damit nicht leicht ängstliche aber sonst vielleicht sehr brauchbare Pflegepersonen abgeschreckt und dem Pflegerberufe entfremdet werden. Wie ein Einblick in die vorhandenen Bestimmungen zeigt, sind namentlich in außerdeutschẹn Ländern die Examenvorschriften zum Teil sehr streng. In Holland, England, Niederösterreich, Frankreich und den Vereinigten Staaten zerfällt die Prüfung in einen schriftlichen und einen mündlichen Teil mit praktischen Ubbungen. In Frankreich findet die schriftliche Prüfung unter großen Kautelen gegenüber Betrugsversuchen statt; in Holland wird jeder Kandidat eine Stunde lang geprüft, und die gestellten Fragen sind oft nicht leicht. So wird, wie Sie mens erwähnt, nach der Zusammensetzung der grauen Substanz gefragt, nach dem Unterschied zwischen Halluzination und Illusion $\mathrm{u}$. dgl.

Die Prüfung findet in den eben bezeichneten außerdeutschen Ländern vor einer besonderen Prüfungskommission statt, welche aus 3-5, in Frankreich sogar aus 10 Mitgliedern besteht; und in Amerika schließt sich an die Prüfung ein Festmahl an, an welchem Zöglinge und Ärzte teilnehmen. Beim Studium aller dieser Einrichtungen hat man oft den Eindruck, als ob hie und da doch etwas zu weit übers Ziel hinausgeschossen wird, wenngleich ich gern zugebe, daß manches durch die ganze Organisation des Pflegerberufes in den bezeichneten Ländern erklärt sein mag. Unsere Verhältnisse zwingen uns vor der Hand noch, die Prüfung möglichst einfach und nicht zu schwierig zu gestalten, 
sie ist am besten in das Gewand einer Wiederholung zu kleiden; dafür müssen wir aber unbedingt daran festhalten, daß auch tatsächlich jeder Pfleger sich ihr unterzieht. Wer auch unter solchen milden Bedingungen noch davor zurückschreckt, der eignet sich überhaupt nicht zum Pflegerberuf, denn allzu zaghafte Naturen ohne jedes Selbstvertrauen können wir nicht gebrauchen.

Eine besondere Prüfungskommission erscheint vorläufig nicht erforderlich; sie macht unnötige Kosten und setzt doch vor allem voraus, daß das ganze Unterrichtswesen einheitlich geregelt ist, wovon wir ja noch weit entfernt sind. Bei uns wird die Prüfung vom Direktor und dem unterrichtenden Arzt abgehalten. Sie ist nur mündlich und erstreckt sich sowohl auf den theoretischen als auf den praktischen Teil der allgemeinen Krankenpflege und der Jrrenpflege. Zugrunde gelegt wird im allgemeinen der Scholzsche Leitfaden.

Ưber das Ergebnis der Prüfung erhält jeder Prüfling eine Note, die in seinen Personalakten vermerkt wird. Die Teilnahme am Kursus wird unter Beifügung der empfangenen Note in der Regel nur beim Austritt aus dem Dienste im Abgangszeugnisse bescheinigt. Besondere Diplome, wie sie in England, Holland und anderen Ländern, dagegen in Deutschland soviel ich weiß noch nirgends üblich sind, werden auch bei uns nicht erteilt. Dagegen ist zu erwägen, ob man nicht durch Aussetzen kleiner Prä mien (Bücher usw.) für die besten Leistungen den Lerneifer noch erhöhen könnte.

Bei Festsetzung der Note ist es erwünscht, daß hierbei nicht der Ausfall der Prüfung allein maßgebend ist, sondern daß daneben auch die Gesamtleistungen mit berücksichtigt werden, denn das Ergebnis einer Prüfung hängt ja, wie ich nicht erst hervorzuheben brauche, von zu vielen Zufälligkeiten ab und gibt durchaus nicht immer ein richtiges Bild von den Kenntnissen und der Brauchbarkeit des einzelnen. Erst dann, wenn die Note unter Einbeziehung der Gesamtleistungen erteilt wird, darf sie für die Erlangung gewisser Vorteile (Gehaltsaufbesserung, Aufrücken in bessere Stellen usw.) maßgebend sein. Dann ist es aber auch dringend erwünscht, $d a ß$ sie grundsätzlich jedem austretenden Pfleger in das Abgangszeugnis eingetragen wird, damit er auch anderwärts gleich in der richtigen Weise eingeschätzt werden kann. Die von mancher Seite geäußerte Befürchtung, daß durch Erteilung von Zeugnissen das Umherziehen in den Anstalten begünstigt würde, kann ich nicht voll teilen; ich bin der Meinung, daß bei den sog. „Wandervögeln" der Hauptsache nach doch andere treibende Faktoren im Spiele sind.

Wer bei uns die Prüfung nicht besteht, was im ganzen selten vorkommt, ist gehalten, am folgenden Kursus nochmals teilzunehmen. Doch ziehen es die „Durchgefallenen" in der Regel vor, freiwillig aus 
dem Anstaltsdienst zu scheiden. Eine abermalige Teilnahme an einem Kursus nach Verlauf von 1-2 Jahren empfiehlt sich aber auch für diejenigen, welche die Prüfung bestanden haben, zur Wiederauffrischung ihrer Kenntnisse. Solche Wiederholungskurse bestehen, wie ich aus den Berichten entnehme, schon in verschiedenen Anstalten. Starlinger konnte sie in 16 Anstalten feststellen.

Die günstigste Jahreszeit für die Abhaltung des Pflegerunterrichtes sind wohl überall die Wintermonate, in denen die Feldarbeiten ruhen, und eine längere Beurlaubung von Pflegepersonen nur selten stattfindet. Es besteht aber bei uns die Gepflogenheit, dann, wenn im Frühjahr der Personalwechsel ein besonders großer war, im So m meranfang die Neulinge in einem ganz kurzen vorläufigen Kursus wenigstens in den allerwichtigsten Punkten der Krankenpflege zu unterweisen.

Neben diesen obligatorischen Kursen und Wiederholungskursen mit möglichst eingeschränktem. Lehrprogramm sollten an jeder Anstalt von Zeit zu Zeit noch eine Art erweiter ter Kurse stattfinden, an denen teilzunehmen ins Belieben der einzelnen gestellt würde. Diese freiwilligen Kurse müßten in erster Linie der weiteren Ausbildung derjenigen Pflegepersonen dienen, welche sich die Anwartschaft auf die Stelle eines Abteilungspflegers sichern wollen; aber auch abgesehen von diesem Zweck wären sie ein gutes Mittel, um in dem gleichmäßigen Anstaltsbetriebe anregend zu wirken und der leicht einreißenden Versimpelung vorzubeugen. Wenn ich recht unterrichtet bin, besteht eine ähnliche Einrichtung, wie ich mir sie denke, bereits in der Irrenanstalt zu Weinsberg.

Der Lehrstoff müßte in diesen freiwilligen Kursen vor allem eine Erweiterung des in den pflichtmäßigen Kursen Vorgetragenen darstellen. Aus der allgemeinen Krankenpflege wären etwa zu behandeln: Die Wirkung der wichtigsten Arzneien, der Nährwert der Speisen, die Diätetik des Krankenbettes, die Wochenbett- und Säuglingspflege, schwierigere Verbände, die Narkose u. ähnl. Aus dem Gebiete der Irrenpflege wäre etwas Geschichte der Psychiatrie vorzutragen; ferner wären hier die wichtigsten Krankheitsformen, nicht bloß einzelne Symptome, zu besprechen. Sehr empfehlenswert wären auch Utbungen in der Abfassung schriftlicher Krankheitsberichte. Die Berichte müßten dann korrigiert und unter gleichzeitiger Berücksichtigung der Stilistik und Orthographie durchgesprochen werden. (Erwähnt sei an dieser Stelle die bei uns schon bestehende Einrichtung, daß über jede Neuaufnahme von dem betreffenden Abteilungspfleger ein kurzer schriftlicher Bericht zu erstatten ist.) Zur Hebung der Allge meinbildung könnten in diesem erweiterten Kursus noch einige andere Wissenszweige in das Lehrprogramm aufgenommen werden, wobei in erster Linie die Natur- 
wissenschaften zu berücksichtigen wären. Sehr gut eignen würden sich hierzu einige ausgewählte Kapitel aus dem Gebiete der Physik oder der Botanik, z. B. die Besprechung der wichtigsten Giftpflanzen, der eßbaren und giftigen Pilze u. ähnl. Bei der Auswahl des Vorzutragenden würde sich dem Geschmack und der persönlichen Begabung des einzelnen ein weiter Spielraum eröffnen; in jedem Falle wäre es aber dringend nötig, daß auch in diesem erweiterten Kursus der behan delte Lehrstoff nicht völlig den Zusammenhang mit der Kranken pflege verliert. Ich kann mich deshalb auch nicht mit den von einigen Seiten gemachten Vorschlägen befreunden, die Pfleger in der schönen Literatur, in der Mathematik oder gar im Französischen zu unterrichten. Wir wollen uns doch vor allem tüchtige Pfleger heranbilden, sollten dabei aber alles vermeiden, die Halbbildung, die sich ohnehin schon überall breit genug macht, auch noch durch unser $\mathrm{Zu}$ tun $\mathrm{zu}$ fördern.

$\mathrm{Zu}$ den Mitteln, das Personal fortzubilden und berufstüchtig zu erhalten, gehört auch, daß nicht bloß die jüngeren, sondern auch die älteren Pfleger, und zwar namentlich die Stationspfleger in regelmäßigem Wechsel den Dienst auf den verschiedensten Abteilungen übernehmen. Gerade die Stationspfleger kommen dadurch, daß sie zuweilen viele Jahre hindurch immer derselben, manchmal recht bequemen Abteilung vorstehen, leicht in Gefahr, rückständig zu werden. Es empfiehlt sich daher sehr, daß sie von Zeit zu Zeit die Abteilungen untereinander austauschen; gelegentlich der Bestandsaufnahme am Jahresschlusse läßt sich das ohne große Schwierigkeiten bewerkstelligen.

Auf einen Punkt möchte ich hier noch mit einigen Worten eingehen, der auch in gewisser Beziehung zur Ausbildung des Personals steht. Es liegt der Gedanke sehr nahe, und er ist auch schon ausgesprochen worden, daß wir uns durch eine möglichst gründliche Erziehung unseres Personals heimliche Kurpfuscher heranbilden. Nach meinen Erfahrungen haben wir aber keinen Anlaß zu einer solchen Befürchtung. Ich habe schon seit Jahren dieser Frage meine Aufmerksamkeit gewidmet, habe aber nie den geringsten Anhaltspunkt dafür gewinnen können, daß unser Pflegepersonal - auch das nicht mehr im Anstaltsdienst tätige - draußen Kurpfuscherei treibt. $\mathrm{Ob}$ es in anderen Gegenden sich anders verhält, vermag ich allerdings nicht $\mathrm{zu}$ beurteilen.

Die Ausbildung unseres Pflegerpersonals würde sich scheinbar viel einfacher gestalten, wenn man von vornherein nur sog. gebildete Personen als Pfleger anstellte. Diese Forderung wird ja von verschiedenen Seiten erhoben, und es soll diese Maßnahme einen Fortschritt auf dem Gebiete der Irrenpflege bedeuten. Ich muß gestehen, daß ich 
noch nie das Bedürfnis nach solchem gebildeten Personal empfunden habe. Bei uns stammen die Pfleger und Pflegerinnen der Hauptsache nach aus den gleichen Volksschichten wie die Kranken selbst (abgesehen von den wenigen Pensionären), und die hierdurch bedingte Übereinstimmung der Lebensanschauungen und Gemeinsamkeit der allgemeinen Interessen, welche die geistige Annäherung und das gegenseitige Verstehen wesentlich erleichtert, halte ich bei dem ständigen Zusammenleben zwischen Kranken und Pflegern eher für einen Vorzug als für einen Nachteil. Bei Ausübung der Krankenpflege und besonders der Irrenpflege kommt es doch nicht vor allem auf ein möglichst großes allgemeines Wissen an, nein, Herzengüte und ein lauterer Charakter sind die Eigenschaften, welche in erster Linie zum Pflegerber uf geeignet machen, und diese finden wir in den sogenannten gebildeten Kreisen wirklich nicht häufiger als in den mittleren Volksschichten. Wenn diese Eigenschaften von Hause aus mitgebracht werden, dann kommt es nur noch darauf an, daß das uns zugewiesene Personal etwas bildungsfähig ist; dann werden wir bei einiger Mühe aus ihm auch sicher etwas Brauchbares machen und ihm getrost unsere Kranken anvertrauen können. An Bildungsfähigkeit mangelt es aber der Mehrzahl unseres Personals sicher nicht. Dies gibt selbst Scholz, der besonders eindringlich verlangt, ,daß die Irrenpflege nur in den Händen gebildeter weiblicher Elemente liegen müßte", zu, indem er sagt: „Halte man doch die unteren Schichten nicht für so unbegabt und stumpf". Forel hat einmal den Ausspruch getan: zu einem guten Wärter gehört vor allem Geduld, Gutmütigkeit, sogar etwas Beschränktheit. Wenn auch die letzten Worte nicht ohne jede Einschränkung zu verstehen sind, so liegt doch ein wahrer Kern darin. Ich erinnere mich noch recht gut eines Pflegers, der am Schluß des Kursus, noch wenige Tage vor der Prüfung, auf die Frage nach den Hauptbestandteilen des menschlichen Körpers die Antwort gab: „Der Mensch besteht aus Kopf, Bauch und Beine." Zu den intelligenten Leuten gehörte dieser Mann sicher nicht, aber ein guter Pfleger war er doch. Er versah seinen Dienst mit großer Geduld und Gewissenhaftigkeit, und ich wußte die Kranken bei ihm wirklich gut aufgehoben. Für ein unbedingtes Erfordernis halte ich also gebildetes Personal nicht, wenigstens nicht für unsere öffentlichen Anstalten (für Privatanstalten mag die Sache anders liegen), ich möchte mich fast sogar dagegen aussprechen aus verschiedenen Gründen, auf die näher einzugehen uns hier zuweit führen würde. Utbrigens scheint in den gebildeten Kreisen die Neigung für den Irrenpflegeberuf gar nicht sehr groß zu sein, was sich schon daraus ergibt, daß bei Ausschreibungen von Pflegerinnenstellen niemals Bewerbungen aus diesen Kreisen eingehen.

Bei der Behandlung der Pflegerfrage kehren immer und immer 
die Klagen über den zu häufigen Wechsel des Personals wieder, und dieser wohl überall vorhandene Mißstand ist sicherlich geeignet, die Begeisterung für die regelmäßigen Pflegerkurse allmählich abzuschwächen. Allein wir müssen uns, wie Deiters sagt, ,durch gute Erfolge bei einzelnen über die vielen Mißerfolge trösten lassen“. Um den aus dem ständigen Personalwechsel sich ergebenden dienstlichen Schwierigkeiten abzuhelfen, hat man wiederholt die Errichtung von Pflegerschulen vorgeschlagen, wie sie im Königreich Sachsen schon seit längerer Zeit bestehen. Es erscheint mir aber unsicher, ob dieses wirklich der richtige Weg zu einer befriedigenden Lösung dieser schwierigen Frage wäre. Zunächst ist hierdurch auch noch keine Sicherheit für einen ausreichenden Ersatz der ausscheidenden Pfleger gegeben, denn wie die sächsischen Berichte gerade der letzten Zeit besagen, vermögen die vorhandenen Pflegerschulen den Bedarf nicht immer zu decken. Ferner würde, solange die Irrenfürsorge eine innere Angelegenheit der Provinzen ist, die Errichtung solcher Schulen eine nicht unerhebliche finanzielle Mehrbelastung der einzelnen Provinz bedeuten, selbst dann, wenn diese Schulen nicht selbständig wären, sondern an eine Irrenanstalt angegliedert würden. In letzterem Falle würde aber wieder eine Quelle für unliebsame Rangstreitigkeiten geschaffen sein, indem die Anstalten mit Pflegerschulen sich gegenüber den anderen Anstalten herausgehoben fühlen würden. Aus allen diesen Gründen scheint es mir das beste, daß zunächst noch weiter an der alten Gepflogenheit festgehalten wird, wonach jede Anstalt sich ihr Personal selbst heranbildet, eine Anschauung, die auf den Versammlungen des Vereins deutscher Irrenärzte zu Heidelberg (1896) und Hannover (1897) ziemlich allgemein und bestimmt zutage trat. Wohl aber würde es sich empfehlen, wenn schon jetzt wenigstens innerhalb der einzelnen Provinzen die Bestimmungen über den Pflegerunterricht einheitlich geregelt würden. Für eine Errichtung von Pflegerschulen wird meines Erachtens der geeignete Zeitpunkt erst dann gekommen sein, wenn das Irrenwesen einmal verstaatlicht werden sollte. Dann könnte auch die Reform noch weiter um sich greifen und zu einer Erfüllung anderer zweckmäßiger Forderungen wie die Einsetzung besonderer Prüfungskommissionen, Diplomierung der Geprüften usw. führen.

Zum Schluß noch einige Worte über die durch den Pflegerunterricht erzielten Resultate. Abgesehen von ganz wenigen Ausnahmen stimmen alle Berichterstatter darin überein, daß der Erfolg ein befriedigender ist. Dieser Erfolg läßt sich natürlich, wie Mercklin richtig sagt, nicht ziffernmäßig angeben, aber die Erfahrung zeigt uns doch, daß wir jetzt, wo wir der Ausbildung des Personals immer mehr Aufmerksamkeit schenken, an ihm eine viel bessere Hilfe und Stütze 
bei der Behandlung der Kranken haben als früher. Fischer hat daher ganz recht, wenn er Salgo gegenüber die bessere Ausbildung des Personals als eine elementare Notwendigkeit bezeichnet. Leider haben die Pflegerkurse entgegen früheren Erwartungen einen günstigen Einfluß auf den übermäßigen Personalwechsel nicht gezeigt. Unter den Mitteln, welche früher zur Bekämpfung des ständigen Pflegermangels vorge. schlagen wurden, standen immer obenan eine höhere Besoldung und eine gründliche Ausbildung. Nach beiden Richtungen ist im Laufe der Zeit schon manches besser geworden, aber die Klagen über häufigen Personalwechsel sind nicht verstummt, wenigstens nicht hinsichtlich der Pflegerinnen. Bei den Pflegern macht sich dort, wo die Lohn- und Anstellungsverhältnisse günstiger geworden sind, immer mehr die Neigung geltend, dem Beruf dauernd treu zu bleiben; bei den Pflegerinnen dagegen hat auch die Aufbesserung der materiellen Lage nicht viel geholfen. Einige von ihnen bleiben wohl dem Berufe erhalten und bilden dann einen sehr brauchbaren alten Stamm; die meisten aber scheiden schon nach verhältnismäßig kurzer Zeit wieder aus, entweder weil ihnen die straffe Anstaltszucht nicht behagt oder hauptsächlich weil sie heiraten wollen. Dies ist ganz natürlich und wird wohl zunächst auch weiterhin so bleiben. Der Zukunft bleibt die schwere aber dankbare Aufgabe vorbehalten, Mittel und Wege für die Schaffung eines Standes von wirklichen Berufspflegerinnen zu finden.

\section{Literaturverzeichnis.}

Bresler, Bericht über den gegenwärtigen Stand des Irrenwesens. Psych.-Neurol. Wochenschr. 1908/1909, S. $255 \mathrm{ff}$.

Deiters, Der Stand des Irrenewsens innerhalb des deutschen Sprachgebietes im Jahre 1900/1901. Psych.-Neurol. Wochenschr. 1902/1903, S. 237 ff.

- Über Fortschritte des Irrenwesens. Psych.-Neurol. Wochenschr. 1903/1904, S. 144 und 1904/1905, S. 411.

van Erp Taalman Kip, Das holländische Irrenwesen. Psych.-Neurol. Wochenschrift 1899/1900, S. $72 \mathrm{ff}$.

Hocka uf, Studienreise zum Besuche der Irrenanstalten in den Vereinigten Staaten Nordamerikas. Psych.-Neurol. Wochenschr. 1906/1907, S. $399 \mathrm{ff}$.

Ho p pe, Zur Wärterfrage. Allgem. Zeitschr. f. Psych. 54, S. $644 \mathrm{ff}$.

Mercklin, Der Unterricht des Irrenpflegepersonals. Allgem. Zeitschr. f. Psych. 53.

- Zum Fachunterricht des Irrenpflegepersonals. Psych.-Neurol. Wochensohr. 1900/1901, S. 169.

Scholz, Die Ausbildung des Pflegepersonals. Psych.-Neurol. Wochenschr. 19081909 , S. $409 \mathrm{ff}$.

Siemens, Weitere Vorschläge zur Wärterfrage. Allgem. Zeitsohr. f. Psych. 54, S. $898 \mathrm{ff}$.

Starli nger, Zum gegenwärtigen Stande der Pflegerfrage. Psych.-Neurol. Wochenschrift 9, S. $325 \mathrm{ff}$. 
Stransky, Zur Erziehung und Organisation des Irrenpflegepersonals. Psych.Neurol. Wochenschr. 1906/1907, S. 251 ff.

Werner, Über Fortschritte des Irrenwesens. Psych.-Neurol. Wochenschr. 19111912, S. $367 \mathrm{ff}$.

Wickel, Einiges zur Pflegerfrage. Psych.-Neurol. Wochenschr. 1907/1908, S. 125.

Fuhrmann, Über Bildung unseres Pflegepersonals. Psych.-Neurol. Wochenschr. $1905 / 1906$, S. $313 \mathrm{ff}$.

Landeskongreß der Ungarischen Irrenärzte in Budapest (26. 10. 1902). Psych.-Neurol. Wochenschr. 1902/1903, S. 451.

Bericht der Examenskommission der niederländischen Gesellschaft für Ps ychiatrie über das Jahr 1895 und 1896. Allgem. Zeitschr. f. Psych. 55. S. 41 . 